\section{Incidencia, tipología y medidas de prevención y tratamiento de las lesiones cutáneas asociadas al uso de EPI en profesionales sanitarios durante la pandemia de COVID-19 en España}

Estudio colaborativo GNEAUPP-Cátedra de estudios avanzados en heridas GNEAUPP - FSJJ - Universidad de Jaén
J.E. Torra-Bou* , J.J. Soldevilla-Agreda, F.P. García-Fernández, J. Verdú-Soriano, M. Rodríguez-Palma, J.F. Jiménez-García, J.A. Esperón-Güimil, C. Blasco-García, P. Ibars-Moncasí, J. Rueda-López, E. Perdomo-Pérez, T. Segovia-Gómez, L. Arantón-Areosa, P. López-Casanova, F. Martínez-Cuervo y P.L. Pancorbo-Hidalgo

Miembros del Comité Director del Grupo Nacional para el estudio y Asesoramiento en Úlceras por presión y Heridas Crónicas (GNEAUPP).

*Autor para correspondencia.

Correo electrónico: jetorrabou@hotmail.com (Dr. J.-E. Torra i Bou).

Recibido el 11 de diciembre de 2020; aceptado el 1 de febrero de 2021

\section{Incidence, typology and measures of prevention and treatment of skin lesions associated with the use of personal protective equipment in health care professionals during the COVID-19 pandemic in Spain}

Collaborative study GNEAUPP-Cátedra de estudios avanzados en heridas GNEAUPP - FSJJ - Universidad de Jaén

\section{RESUMEN}

Introducción: La reciente pandemia de COVID-19 ha representado la utilización por parte de los profesionales de la salud de diferentes equipos de protección individual (EPI), lo que ha dado lugar a la aparición de lesiones cutáneas asociadas a los mismos (LC-EPI). Conocer la epidemiología, las características y los factores relacionados con el uso de los diferentes tipos

de EPI y la prevención y el tratamiento de las LC-EPI puede ser muy útil para comprender el alcance del problema y definir estrategias para su prevención y tratamiento en posibles pandemias futuras. Métodos: Estudio transversal realizado por el Grupo Nacional para el Estudio y Asesoramiento

en Úlceras por Presión y Heridas Crónicas (GNEAUPP) en el ámbito geográfico del Estado español, mediante una encuesta autoadministrada utilizando un cuestionario de Google Forms. El universo de estudio fueron profesionales de la salud, de cualquier disciplina y nivel asistencial o tipo de institución que habían estado en contacto con pacientes o sospechosos de COVID-19 y habían utilizado EPI durante la primera ola de la pandemia de COVID-19 en España. Resultados: Se obtuvieron 2078 cuestionarios, el 75,5\% de enfermeras, el 10,79\% de técnicos en cuidados auxiliares de enfermería (TCAE) y el 6,6\% de médicos. De los respondedores, el 84,7\% eran mujeres y el 15,3\% hombres con una edad media de 43,7 años.

En el 18,2\% de los casos, la institución del profesional contaba con un protocolo para la prevención de las LC-EPI, en el 10,2\% el profesional no sabía si existía y en el 71,3\% restante la institución no contaba con dicho protocolo. El 29,9\% de los profesionales utilizaba siempre algún producto de prevención de LC-EPI y el 25,3\% en ocasiones.

El 52,4\% de los encuestados informó haber presentado al menos una LC-EPI, de los cuales el 39\% había presentado una, el 35,8\% dos, el 14,3\% tres, el 2,2\% cuatro y el 8,6\% más de cuatro. En relación con el tipo de

\section{ABSTRACT}

Introduction: The recent COVID-19 pandemic has represented the use by health care professionals (HCP) of different personal protective equipment (PPE), resulting in the appearance of skin injuries associated with PPE (PPE-SI). Knowing the epidemiology, characteristics and factors related to the use of different types of PPE and the prevention and treatment of PPE-SI can be very useful to understand the scope of the problem and to define strategies for its prevention and treatment in possible future pandemics. Methods: To this end, the GNEAUPP proposed the performance of a cross-sectional study, in the geographical area of the Spanish state, by means of a self-administered survey using a Google forms questionnaire. The study universe was HCP, from any discipline and from any level of care or type of institution with patients who have been in contact with COVID-19 patients or COVID-19 suspects and have used PPE during the first wave of the COVID-19 pandemic in Spain. Main results: We obtained 2078 questionnaires, $75.5 \%$ from nurses, $10.79 \%$ from nurse aids, and $6.6 \%$ from physicians. Of these, $84.7 \%$ were women and $15.3 \%$ were men with an average age of 43.7 years. In $18.2 \%$ of the cases the institution of the professional had a protocol for the prevention of PPE-SI, in $10.2 \%$ the professional did not know if it existed or not and in the remaining $71.3 \%$ the institution did not have such a protocol. $29.9 \%$ of the professionals always used some prevention product and $25.3 \%$ sometimes. $52.4 \%$ of respondents reported having submitted at least one PPE-SI, of which $39 \%$ had submitted one, $35.8 \%$ two, $14.3 \%$ three, $2.2 \%$ four and $8.6 \%$ more than four. In relation to PPE-SI, 74.3\% were defined as pressure injuries (PI), 11\% were 
lesiones, el 74,3\% fueron definidas como lesiones por presión (LPP), el $11 \%$ como lesiones por fricción (LF), el 8,5\% como lesiones combinadas o multicausales (LCMC) y el 6,1\% como lesiones cutáneas asociadas a la humedad (LESCAH). La duración media de las lesiones incidentes fue de 11,6 días (9,7 días para las LPP, 10,2 días para las LF, 19,9 días para las LCMC y 19,4 días para las LESCAH). En el artículo se presenta información más detallada por tipo de dispositivo causante, tipología y gravedad de las lesiones por dispositivo, así como las medidas preventivas utilizadas.

PALABRAS CLAVE: COVID-19, equipo de protección individual, lesiones por presión, lesiones cutáneas, lesiones cutáneas relacionadas con la dependencia, prevención, tratamiento, epidemiología, profesionales de la salud, España. friction injuries (FI), 8.5\% were combined or multicausal injuries (CMCI) and 6.1\% were skin injuries associated with moisture (MASI). The mean duration of incident injuries was 11.6 days (9.7 days for PI, 10.2 days for FI, 19.9 days for CMCI and 19.4 days for MASI). More detailed Information is presented in the paper by type of device causing, typology and severity of injuries per device as well as preventive measures used.

KEYWORDS: COVID-19, Personal protective equipment, Pressure injuries, Skin injuries, Dependence skin related injuries, Prevention, Treatment, Epidemiology, Health Care Professionals, Spain.

\section{$\searrow$ INTRODUCCIÓN}

Los tiempos que estamos viviendo, marcados en gran medida por la pandemia de COVID-19, han puesto de manifiesto la vulnerabilidad de los sistemas sanitarios, desde los más a los menos avanzados. La sociedad en su conjunto ha tenido que adaptarse, rápidamente, a esta nueva situación, y el sistema sanitario en especial.

Todo el mundo se hace eco de los efectos devastadores de esta pandemia, y parece que los hasta ahora principales problemas de salud han pasado a un segundo plano.

Las lesiones cutáneas relacionadas con la dependencia (LCRD), como las lesiones por presión (LPP), ya sean por el apoyo de los pacientes en decúbito supino, prono o sedestación o las asociadas a dispositivos clínicos (LPP-DISCLIN) como mascarillas, sondas, catéteres, circuitos de ventilador u otro tipo de dispositivos clínicos en contacto directo con los pacientes, han sido un problema en primera línea en el transcurso de la atención a pacientes con COVID.

La pandemia de COVID-19 ha puesto de manifiesto la necesidad de que los profesionales de la salud hayan tenido que protegerse con los denominados equipos de protección individual (EPI). La utilización de los EPI por parte de los profesionales de la salud se ha traducido en la aparición de lesiones cutáneas, las denominadas lesiones cutáneas asociadas a EPI (LC-EPI), que en muchas ocasiones comparten factores etiológicos o causales con las LCRD, en este caso, en personas no dependientes como los profesionales sanitarios. Aunque muchas de las LC-EPI son LPP-DISCLIN, no se pueden asociar de manera automática ambos términos.

En este sentido podemos hablar de una pandemia silente y desconocida que ha sido definida por los profesionales sanitarios como "la epidemia bajo las sábanas" y que hoy ha trascendido de las sábanas y ha pasado a estar también en la piel de los profesionales que los atienden debajo de los EPI.

La dimensión epidemiológica de esta asociación entre LRCD y la pandemia de COVID-19 es poco conocida y algunos autores como Tomas Vecina et al. $2020^{1}$ se atreven a estimar que el impacto de las LPP, consideradas como un evento adverso en el sistema sanitario durante la primera ola de la pandemia, se ha visto incrementado en un 388\% (atrás queda el estudio ENEAS en el que se recogía que el 7,6\% de los eventos adversos estaban relacionados con los cuidados, y dentro de estos un $3,7 \%$ correspondía a las LPP) ${ }^{2,3}$.

El impacto en los profesionales sanitarios está por determinar, pero los estudios epidemiológicos realizados en China estiman que las LCEPI han pasado de ser imperceptibles hasta afectar a más del 70\% de los profesionales sanitarios. No cabe duda de que se hace necesario evidenciar de forma urgente el impacto devastador que está provocando esta pandemia en las personas mayores y en aquellos que están siendo atendidos y atendiendo en los centros sanitarios y sociosanitarios de todo el mundo. Los resultados se han de analizar basándose en los factores etiológicos que inciden en esta tipología de lesiones, de modo que se podrían identificar como sigue.

\section{TIPOS Y ETIOLOGÍA DE LAS LESIONES EN TIEMPOS DE COVID-19}

\section{Lesiones cutáneas relacionadas con la dependencia (LCRD) ${ }^{4}$}

En el año 2014, García-Fernández et al..$^{5}$ desarrollaron un nuevo marco teórico que explicaba el origen de las LPP y otras lesiones cutáneas relacionadas con la dependencia. Marco que aún hoy sigue en evolución. En este sentido, las personas con cierto grado de dependencia, ya sea funcional o psíquica, que no se pueden desenvolver por sí solas, tienen un mayor riesgo de desarrollar lesiones en la piel y los tejidos subyacentes por diferentes factores causales bien diferenciados.

Así, las personas que por su situación se encuentran sometidas a presión o la combinación de presión y cizalla pueden desarrollar LPP, mientras que aquellas que se encuentran sometidas a un exceso de humedad (orina, heces, saliva, etc.) podrán desarrollar lesiones por humedad (LESCAH). Del mismo modo, aquellas que están expuestas a las fuerzas de fricción (arrastre en la superficie de la cama, movimiento repetido de los talones en contacto con una superficie, etc.) pueden desarrollar lesiones por fricción. Posiblemente, junto a estas lesiones ya claramente identificadas se pueden ańadir otras como la aparición de lesiones cutáneas al final de la vida o lesiones que aparecen en personas con compromiso vital grave. Y en este grupo de lesiones, en general, se encuentran todos aquellos que antes de la pandemia ya tenían estos riesgos, más los que se añaden por estar convalecientes tras haber contraído la COVID-19. En resumen, un gran número de personas con lesiones han trasladado a los sistemas sanitarios un problema y reto de gran dimensión, tanto por el incremento del problema como por la necesidad de recursos específicos para abordar este acontecimiento sanitario y social. Tal como expuso David en marzo de $2020^{6}$, las fases de las epidemias van avanzando: en primer lugar se cobran vidas; en la segunda fase se exigen responsabilidades y recursos, pero no solo para luchar contra la pandemia, sino 
para luchar contra aquellos problemas ya existentes previamente y que han emergido por ser parte de un problema poco considerado en las agendas de política sanitaria y que conduce a una tercera fase que lleva a la sociedad a pedir explicaciones, que según este autor coincide con un pánico generalizado de los ciudadanos. Apunta que esta fase puede ser tan dramática y perturbadora como la propia pandemia.

\section{Lesiones por presión relacionadas con el uso de dispositivos clínicos (LPP-DISCLIN) 7,8}

Es sabido que debido a la situación de salud de algunas personas deben hacer uso de algunos dispositivos clínicos aplicados con fines diagnósticos o terapéuticos. Este hecho hace que el tejido sometido a presión bajo estos dispositivos, que puede ser la piel o los tejidos subyacentes o también mucosas como la uretra, la boca o el oído, entre otros, pueda desarrollar LPP, cambios en el microambiente (humedad, temperatura) o fricción, por exposición a estos dispositivos.

Este tipo de lesiones suelen darse en la piel subyacente al dispositivo, adquiriendo su forma, y deben clasificarse siguiendo el sistema de clasificación de las LPP. Por otro lado, cabe destacar también las LPP en la membrana mucosa, las cuales también están relacionadas con el uso de dispositivos clínicos. En los últimos años, el Grupo Consultivo Nacional Americano de Úlceras por Presión (NPIAP) ${ }^{9}$ ha reconocido que las LPP-DISCLIN son cada vez más frecuentes en todos los entornos de atención y en todos los grupos de edad. No obstante, los grupos más vulnerables son los pacientes críticos, neonatos y niños, adultos mayores y pacientes bariátricos.

Es importante distinguir las lesiones relacionadas con la inmovilidad de las asociadas a dispositivos. Las relacionadas con la inmovilidad ocurren con frecuencia sobre una prominencia ósea o tejido expuesto a la presión de una superficie de soporte, y las asociadas a los dispositivos, a menudo reflejan la forma y ubicación de un dispositivo clínico. Este tipo de lesiones pueden ser más complicadas de prevenir, puesto que el dispositivo puede ser un componente esencial del tratamiento?.

Un reciente documento de consenso elaborado por un panel de expertos $^{10}$ define las lesiones asociadas a los dispositivos clínicos como "Device Related Pressure Ulcers" [úlcera por presión relacionada con dispositivos (UPRD)], para poner énfasis en que puede haber dispositivos que den lugar a estas lesiones mientras están aplicados con fines diagnósticos o terapéuticos. Así, indican "que una UPRD implica una interacción con un dispositivo u objeto que está directa o indirectamente en contacto con la piel... o implantado bajo la piel, causando fuerzas focales y locales que deforman los tejidos superficiales o subyacentes más profundos".

\section{Lesiones cutáneas asociadas a los equipos de protección individual (LC-EPI) y lesiones cutáneas asociadas a los métodos de higiene y antisepsia de manos (LC-HA)}

En realidad, estas lesiones no difieren de las ya mencionadas anteriormente, sino que, a diferencia del factor etiológico, que será el mismo, lo que varía es a quién afecta. En este caso, a los profesionales, sanitarios o no, que tienen que hacer uso de EPI y estar sometidos a un lavado de manos o antisepsia de estas, de manera frecuente.

En el caso del uso de EPI, nos encontramos con el riesgo de desarrollar lesiones cutáneas asociadas al uso de dispositivos que ejercen presión sobre nuestros tejidos y además modifican el microambiente, aumentando la humedad y la temperatura a nivel local. Estos son: uso de mascarillas, gafas de protección, pantallas de protección, guantes, etc.
En el caso de la higiene y/o antisepsia, y el uso de guantes, esto hace que se modifiquen las características de la piel y sus funciones. Principalmente, se altera el manto lipídico y el $\mathrm{pH}$ de la piel, lo que aumenta el riesgo de dermatitis y posibles lesiones asociadas.

\section{Lesiones cutáneas como manifestación de COVID-19 (LC-COVID)}

Se postula que el coronavirus que produce esta pandemia puede tener manifestaciones a nivel cutáneo, y en la literatura científica se han presentado casos de acroisquemia ${ }^{11,12}$, principalmente, en niños o jóvenes. También se han presentado algunas manifestaciones cutáneas como exantemas cutáneos, urticaria, vesículas o vasculitis ${ }^{13,14}$.

Los profesionales sanitarios que nos dedicamos a la prevención y el tratamiento de heridas nos encontramos, actualmente, ante un doble reto: doblegar y aplanar la curva de esta terrible pandemia y a la vez prevenir que la pandemia "bajo las sábanas y los equipos de protección individual" deje de serlo también.

En el caso de las LC-EPI y las lesiones cutáneas por higiene o antisepsia (LC-HA) existen ya algunos artículos que citan este tipo de lesiones en la literatura, a raíz de la pandemia, y cómo afectan a los profesionales sanitarios.

Así, Jiang et al. ${ }^{15}$, en febrero de 2020 llevaron a cabo un estudio transversal mediante un cuestionario on-line para conocer la prevalencia, las características y las medidas preventivas para lesiones de piel en trabajadores sanitarios de la región de Wuhan. En una muestra de 4308 encuestados encontraron una prevalencia de lesiones en la piel asociadas a los EPI del 42,8\% [intervalo de confianza (IC) al 95\%: 41,3-44,3\%] siendo, predominantemente, LPP-DC con exposición a la humedad a causa del aumento de transpiración cutánea por el uso de EPI y desgarros de piel (skin tears en inglés) o erosiones, laceraciones, desgarros cutáneos (ERLADESC) en castellano. El sudor, el tiempo de uso del EPI, ser hombre y llevar EPI de nivel 3 fueron los factores de riesgo que se asociaron en mayor medida a las lesiones cutáneas.

Pei et al. ${ }^{16}$, también en China, realizaron un estudio con una encuesta a 489 profesionales sanitarios. En este caso, el 61,8\% manifestó algún nivel de prurito y el 73,1\% sufrió algún tipo de lesión en la piel tales como eritema $(38,8 \%)$, prurito $(22,9 \%)$, flictenas $(13,8 \%)$ pápulas y otras expresiones cutáneas. La mayoría de estas lesiones se registraron en la cara $(47,1 \%)$, seguido por las manos $(27,5 \%)$, extremidades $(15,7 \%)$, tronco $(12,6 \%)$ y el cuerpo completo $(2,3 \%)$. En este estudio, las lesiones también se asociaron a EPI de mayor seguridad y mayor tiempo de uso del equipo de protección individual.

Lan et al. ${ }^{17}$, en otro estudio con 542 individuos, hallaron una prevalencia general de lesiones en la piel del $97 \%$, donde las principales localizaciones dańadas fueron el puente nasal (la más predominante con el 57,62\%), las manos, la nuca y la frente.

Lin et al. ${ }^{18}$ recogieron datos de 376 participantes con la misma metodología de encuesta on-line donde las reacciones adversas de la piel fueron reportadas en un 75,5\% de la muestra con una distribución semejante a los otros estudios mencionados en cuanto a tipos de lesiones y localizaciones.

Altunisik et al. ${ }^{19}$ se centraron en investigar las reacciones adversas de la piel ante la higiene de manos (LC-HA) en Turquía. Los encuestados atribuían las reacciones en la piel, principalmente sequedad, al uso de guantes y a la limpieza con soluciones hidroalcohólicas y antisépticos.

Hadjieconomou ${ }^{20}$, en el Reino Unido, en una muestra de 72 profesionales de la salud, encontró que el 46,5\% presentó irritaciones en las manos relacionadas con el uso de soluciones de higiene y lavado.

$\mathrm{Hu}$ et al..$^{21}$, en una pequeña muestra de cuestionarios identificaron una incidencia de reacciones adversas en la piel del $95,1 \%$ entre los 
que llevaban máscaras N95 (68,9\% lesiones en el puente nasal y 27,9\% prurito facial), $88,5 \%$ relacionada con el uso de guantes de látex (siendo la sequedad de la piel, el prurito y las erupciones cutáneas los más frecuentes) y un $60,7 \%$ entre los que llevaban ropa protectora (refiriendo sequedad de la piel y prurito).

Maliyar et al. ${ }^{22}$, en una muestra de 546 profesionales de la salud, reportan que el $97 \%$ presentó algún efecto adverso relacionado con el uso de EPI, el $83,1 \%$ se localizó en el puente de la nariz y el $78,7 \%$ en las mejillas. Estos autores consideran que la aparición de los efectos adversos relacionados con el uso de EPI tiene una relación directa con los materiales y sustancias como los formaldehídos que se utilizan en su proceso de fabricación.

En otro orden de cosas, Hu et al. ${ }^{23}$, en una amplia muestra de enfermeras, estudiaron, además de las lesiones en la piel, factores relacionados con la ansiedad, burnout, depresión o miedo. Así, se encontró un moderado nivel de burnout y un alto nivel de miedo. Un 94,8\% tuvo una o más lesiones cutáneas, y los resultados de salud mental correlacionaron positivamente con las lesiones cutáneas.

Finalmente, una reciente Scoping Review ${ }^{24}$ resalta que el uso prolongado de EPI se traduce en manifestaciones cutáneas y lesiones (97\%), siendo el puente nasal la localización más afectada (83\%). Además, los profesionales sanitarios experimentan altos niveles de estrés, depresión, ansiedad e insomnio.

Como se puede observar, existe una alta incidencia de lesiones cutáneas, especialmente en la zona facial, por el uso prolongado de mascarillas y también en las manos por el uso de guantes, soluciones antisépticas o lavado frecuente de manos, además de diferentes problemas de salud mental muy relacionados con el miedo a contraer la enfermedad, pero también se ha evidenciado que por tener lesiones cutáneas.

No se han encontrado trabajos en España, u otros países de nuestro entorno, que se dediquen a investigar los efectos que los EPI puedan tener en los profesionales sanitarios, y es por ello, y justo cuando empezábamos a salir del pico de la pandemia, por lo que por parte del GNEAUPP se planteó la realización del primer estudio epidemiológico sobre LC-EPI.

Conocer la epidemiología, las características y los factores relacionados con la utilización de los diferentes tipos de EPI y de la prevención y el tratamiento de LC-EPI puede ser de gran utilidad para conocer el alcance del problema de las LC-EPI en Espańa y definir estrategias para su prevención y tratamiento en posibles pandemias futuras.

\section{$\searrow$ OBJETIVOS}

- Describir las características demográficas de la muestra de profesionales de la salud que en España han utilizado EPI.

- Describir los tipos de EPI utilizados y variables relacionadas con su tiempo de utilización.

- Describir las medidas de prevención de LC-EPI.

- Determinar la frecuencia, tipología y características de las LC-EPI aparecidas durante la reciente pandemia de COVID-19.

- Describir las medidas de tratamiento de las LC-EPI.

- Asociar, cuando ello sea posible, variables relacionadas con el tipo de EPI, población que la ha utilizado, aparición de LC-EPI, tipo de EPI y medidas de prevención y tratamiento.

\section{METODOLOGÍA}

Se realizó un estudio observacional transversal, mediante un cuestionario autoadministrado a través de Google Forms.

\section{Unidad de estudio}

- La población a estudio fueron aquellos profesionales de la salud que desarrollaban su labor profesional en Espańa, ya sea en centros hospitalarios, comunitarios o centros sociosanitarios, de entidad pública, concertada o privada.

- La muestra quedó configurada por aquellos profesionales de la salud que desarrollaban su labor clínica y de gestión en España y que accedieron a cumplimentar la encuesta.

- Criterios de inclusión y exclusión: se incluyó en el estudio a todos los profesionales que respondieron a la encuesta de forma voluntaria y anónima. No hubo criterios de exclusión excepto no responder las variables de obligada cumplimentación en la encuesta.

- Cálculo del tamaño muestral: se consideró que la población a la cual iba destinada la encuesta era infinita, pues agrupaba cualquier tipo de profesional sanitario de todas las provincias del estado espańol, y tampoco se conocía el volumen de respuestas que podrían obtenerse a través de los medios de difusión empleados. Por ello se hizo un cálculo muestral teniendo en cuenta una población infinita, con un $5 \%$ de máxima imprecisión $(\varepsilon=5 \%)$ asumiendo una estimación de prevalencia de problemas de seguridad del $50 \%$ y un nivel de confianza para la estimación del 95\% (error alfa 5\%).

- Método de selección de la muestra: al tratarse de un cuestionario autoadministrado se autoseleccionaron los profesionales que respondieron la encuesta. No se definió inicialmente un tamaño muestral a cubrir.

\section{Variables}

Se consideraron las siguientes variables de estudio:

- Variables sociodemográficas: edad, sexo, comunidad autónoma en la que trabajaban, profesión y tipo de institución.

- Variables relacionadas con la prevención:

- Disponibilidad en la institución de un protocolo específico sobre la prevención de las LC-EPI.

- Utilización de productos para la prevención de las LC-EPI.

- Proveedor de los productos de prevención.

- Tipos de productos utilizados.

- Variables relacionadas con las lesiones:

- Presencia de lesiones.

- Número de lesiones.

- Dispositivos causantes.

- Horas de utilización del EPI al día.

- Horas desde el uso del EPI hasta la aparición de la lesión.

- Días de duración de la lesión.

- Tipo de lesión y gravedad de esta ${ }^{4}$ (tabla 1 ).

- Localización de la lesión.

- Productos utilizados en la prevención.

- Tratamiento utilizado para la curación.

Finalmente se presentaba una pregunta abierta que permitía hacer comentarios o sugerencias sobre la encuesta.

\section{Método e instrumentos para la recogida de datos}

Para la recogida de datos se elaboró un cuestionario autoadministrado utilizando la herramienta en línea Google Forms (accesible en: https:// docs.google.com/forms/d/1DI1T9Ky2lhwsgmOeSyxtv-10BUwijl39Y0-lkiGt_7U/edit). 
Tabla 1. Tipo de lesión y gravedad

\begin{tabular}{|c|c|c|c|}
\hline Lesiones por presión & $\begin{array}{l}\text { Lesiones combinadas } \\
\text { o multicausales }\end{array}$ & Lesiones por fricción & Lesión por humedad \\
\hline $\begin{array}{c}\text { Categoría I } \\
\text { Lesión por presión (LPP) } \\
\text { de categoría I (eritema que no } \\
\text { palidece en piel intacta) }\end{array}$ & $\begin{array}{c}\text { Categoría I } \\
\text { Lesión por presión (LPP) } \\
\text { de categoría I (eritema que no } \\
\text { palidece en piel intacta) }\end{array}$ & $\begin{array}{c}\text { Categoría I } \\
\text { Eritema sin flictena }\end{array}$ & $\begin{array}{l}\text { Categoría 1A } \\
\text { Eritema leve-moderado sin pérdida } \\
\text { de integridad cutánea }\end{array}$ \\
\hline $\begin{array}{c}\text { Categoría II } \\
\text { Lesión en epidermis, dermis }\end{array}$ & $\begin{array}{c}\text { Categoría II } \\
\text { Lesión en epidermis, dermis }\end{array}$ & $\begin{array}{c}\text { Categoría II } \\
\text { Lesión con flictena }\end{array}$ & $\begin{array}{l}\text { Categoría 1B } \\
\text { Eritema intenso sin pérdida } \\
\text { de integridad cutánea }\end{array}$ \\
\hline $\begin{array}{l}\text { Categoría III } \\
\text { Lesión en epidermis, dermis y tejido } \\
\text { celular subcutáneo }\end{array}$ & $\begin{array}{c}\text { Categoría III } \\
\text { Lesión en epidermis, dermis y tejido } \\
\text { celular subcutáneo }\end{array}$ & $\begin{array}{c}\text { Categoría III } \\
\text { Lesión con pérdida de integridad } \\
\text { cutánea }\end{array}$ & $\begin{array}{c}\text { Categoría 2A } \\
\text { Eritema con pérdida de integridad } \\
\text { cutánea (<50\% de la superficie } \\
\text { del eritema) }\end{array}$ \\
\hline $\begin{array}{c}\text { Categoría IV } \\
\text { Lesión hasta estructura articular, } \\
\text { hueso o cartílago }\end{array}$ & $\begin{array}{c}\text { Categoría IV } \\
\text { Lesión hasta estructura articular, } \\
\text { hueso o cartílago }\end{array}$ & & $\begin{array}{c}\text { Categoría 2B } \\
\text { Eritema con pérdida de integridad } \\
\text { cutánea (>50\% de la superficie } \\
\text { del eritema) }\end{array}$ \\
\hline
\end{tabular}

Fuente: Documento Técnico GNEAUPP N ․ㅜ 2. Clasificación y categorización de las lesiones relacionadas con la dependencia4.

Para aumentar la captación, la difusión de este cuestionario se realizó por parte de los miembros del equipo investigador a través de las redes sociales propias y del GNEAUPP.

Los datos fueron volcados desde esta herramienta a una base de datos Excel y posteriormente se analizaron a través del paquete estadístico SPSS V25.0.

\section{Análisis de los datos}

Se realizó un análisis descriptivo de las variables. De esta manera, las variables cuantitativas se analizaron calculando la media, la desviación típica y su intervalo de confianza del 95\% si tenían una distribución normal, y la mediana y los percentiles 25 y 75 si existía distribución asimétrica y las cualitativas como frecuencias y porcentajes.

Posteriormente, se realizó un análisis comparativo de tipo bivariante, relacionando los datos identificativos del profesional respondiente y los datos relativos a la institución en la que el profesional desarrollaba su labor con el resto de las variables sobre el tipo de lesiones, EPI y productos y cuidados de prevención.

\section{Aspectos éticos de la investigación}

Se garantizó la confidencialidad de los datos obtenidos, siendo los datos clínicos relativos a los residentes anónimos y desvinculados de cualquier dato identificativo respetando la Ley Orgánica 3/2018 del 5 de Diciembre de Protección de Datos Personales y garantía de los derechos digitales (LOPDGDD) y por tanto los derechos ARSO-POL sobre acceso, rectificación, supresión, limitación del tratamiento, portabilidad y oposición según la regulación en los artículos 15 a 22 del Reglamento (UE) $2016 / 679^{25}$.

$\mathrm{Al}$ ser un cuestionario autoadministrado a profesionales no se precisó de la autorización de un comité de ética, puesto que con la cumplimentación del mismo se considera tácita la autorización para participar en el estudio; no obstante, un comité de expertos del GNEAUPP no implicado en el diseńo ni ejecución del estudio valoró los aspectos éticos del mismo.

\section{Y RESULTADOS}

\section{Muestra de estudio}

Durante el período de disponibilidad del cuestionario online se obtuvieron 2086 cuestionarios, de los que 8 fueron excluidos, 2 por falta de datos y 6 por ser de otros países; por tanto, la muestra total fue de 2078 cuestionarios, de los cuales 1760 correspondieron a personas de sexo femenino $(84,7 \%)$ y $318(15,3 \%)$ de sexo masculino. La edad media de los respondedores fue de 43,7 años ( $\mathrm{DE} \pm$ 10,8 años; IC95\%: 43,2-44,1).

Por sexos, la edad media de los respondedores de sexo femenino, 43,4 ańos ( $D E \pm 10,7$ años; IC 95\%: 42,9-43,9), fue ligeramente inferior a los de sexo masculino con 45,4 años ( $\mathrm{DE} \pm 11,1$ ańos; IC 95\%: 44,2-46,7).

En la figura 1 y en la tabla 2 se describe la procedencia geográfica de los participantes, así como su profesión.

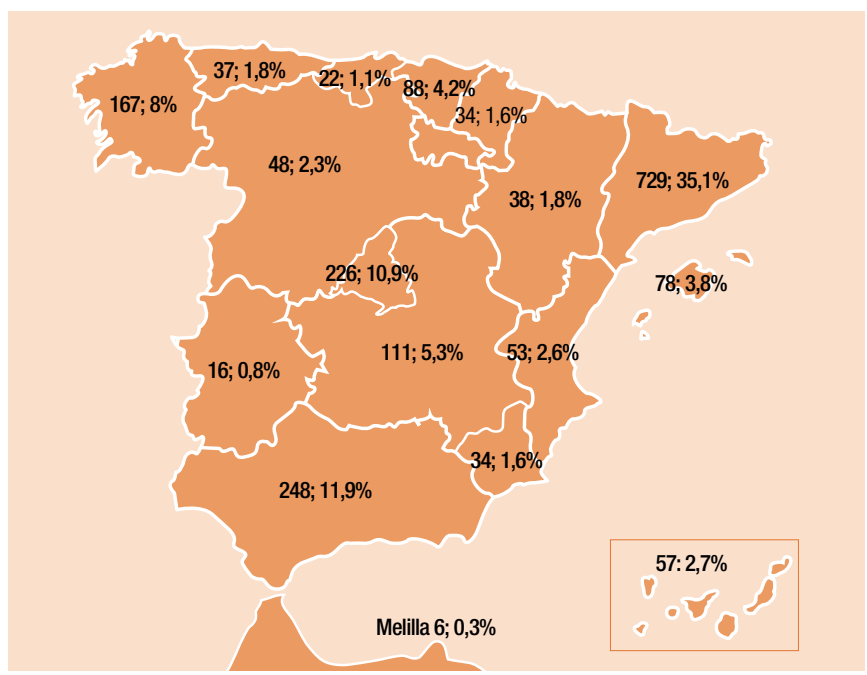

Figura 1. Procedencia geográfica de los participantes. 
Tabla 2. Profesión de los participantes-respondedores

\begin{tabular}{|l|c|c|}
\hline \multicolumn{1}{|c|}{ Profesional } & n & $(\%)$ \\
\hline Enfermera/o & 1611 & 77,5 \\
\hline TCAE & 223 & 10,7 \\
\hline Médica/o & 137 & 6,6 \\
\hline Fisioterapeuta & 26 & 1,3 \\
\hline Celador/a & 23 & 1,1 \\
\hline Técnica/o de laboratorio & 10 & 0,5 \\
\hline Técnica/o de radiología & 9 & 0,4 \\
\hline Administrativas/os - servicios generales & 8 & 0,4 \\
\hline Matronas & 6 & 0,3 \\
\hline Terapeuta ocupacional & 5 & 0,2 \\
\hline Farmacéutica/o & 4 & 0,2 \\
\hline Técnico de mantenimiento/ingeniería & 3 & 0,1 \\
\hline Trabajador/a social & 3 & 0,1 \\
\hline Podóloga/o 0 & 2 & 0,1 \\
\hline Técnica/o de emergencias & 2 & 0,1 \\
\hline Cocina & 1 & 0,05 \\
\hline Limpieza & 1 & 0,05 \\
\hline Logopeda & 1 & 0,05 \\
\hline Odontóloga/o & 1 & 0,05 \\
\hline Psicóloga/0 & 1 & 0,05 \\
\hline Técnica/o radioterapia & 1 & 0,05 \\
\hline
\end{tabular}

TCAE: técnico en cuidados auxiliares de enfermería.

\section{Medidas de prevención de LC-EPI}

Un $18,2 \%$ de los encuestados manifestaron que su institución contaba con un protocolo de prevención de LC-EPI, un 10,5\% no lo sabía y el $71,3 \%$ declaró que su institución no contaba con dicho protocolo. En la figura 2 se muestra la disponibilidad de protocolo según nivel asistencial.

Un total de 621 respondedores $(29,9 \%)$ utilizaban productos para la prevención de LC-EPI, 488 (23,5\%) de ellos los utilizaban con frecuencia o siempre y $969(46,6 \%)$ no los utilizaban.

En la figura 3 se muestra información relativa a quién proporcionaba el material de prevención.

En la tabla 3 se muestran los sistemas o productos para la prevención de LC-EPI utilizados por los profesionales respondedores a la encuesta.

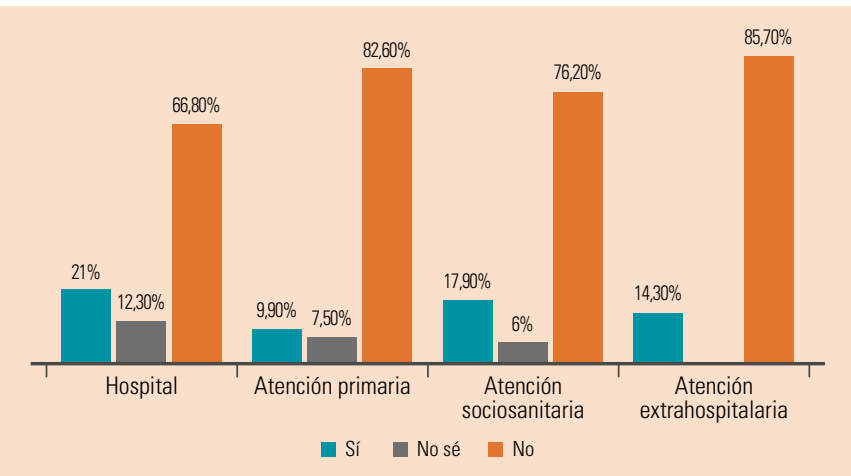

Figura 2. Existencia de protocolo de prevención de LC-EPI.

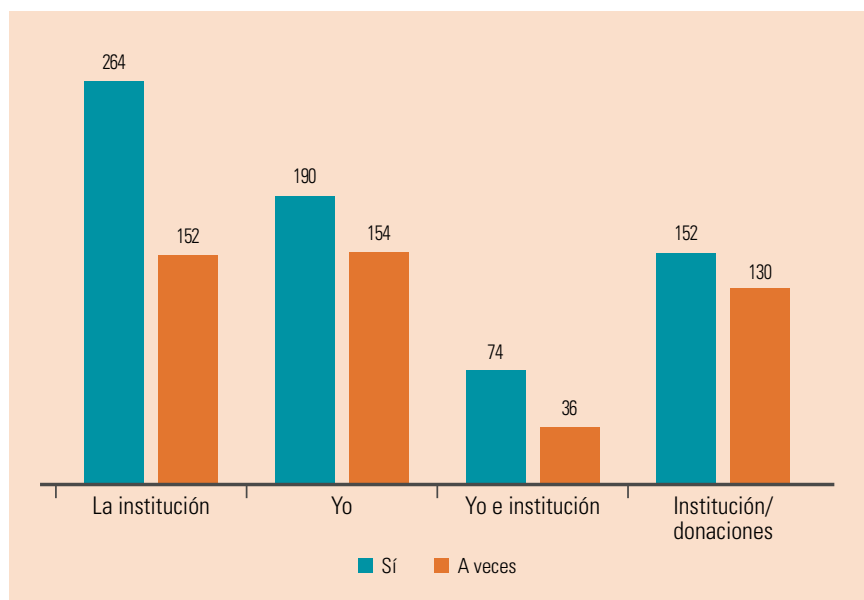

Figura 3. ¿Quién proporciona el material de prevención?

Tabla 3. Sistemas de prevención de LC-EP|a

\begin{tabular}{|l|l|}
\hline \multicolumn{1}{|c|}{ Sistemas de prevención utilizados } & n. \\
\hline Cremas/aceites hidratantes & 815 \\
\hline Apósitos de hidrocoloide & 488 \\
\hline AGHO en aceite & 410 \\
\hline Apósitos de espuma de poliuretano con adhesivo convencional & 220 \\
\hline Apósitos de espuma de poliuretano con adhesivo de silicona & 165 \\
\hline Crema barrera & 136 \\
\hline Apósito de gasa esparadrapo & 121 \\
\hline AGHO en emulsión & 83 \\
\hline PCNI & 75 \\
\hline Apósitos de película de poliuretano & 66 \\
\hline Salva orejas & 23 \\
\hline Sujeta mascarillas/tiras & 15 \\
\hline Tiras adhesivas & 3 \\
\hline Cremas corticoides & 3 \\
\hline Botones & 3 \\
\hline Electrodos recortados & 2 \\
\hline Aceite esencial de lavanda natural & 1 \\
\hline Aceite hipérico & 1 \\
\hline Bálsamo labial & 1 \\
\hline Vaselina líquida & 1 \\
\hline Crema con corticoides & 1 \\
\hline Algodón & 1 \\
\hline Oily milk ozono & 1 \\
\hline Rosa mosqueta & 1 \\
\hline Hidrogel & 1 \\
\hline Gasas & 1 \\
\hline Crema con pantenol (vitamina B5) & 1 \\
\hline Cinta sujeción bolsa de orina & 1 \\
\hline Crema cicatrizante & 1 \\
\hline Crema/mascarilla cutánea & 1 \\
\hline Áloe vera & 1 \\
\hline Jabón hidroalcohólico & 1 \\
\hline Jabón neutro & 1 \\
\hline Malla de silicona & 1 \\
\hline Ropa de algodón con cuello alto & 1 \\
\hline
\end{tabular}

Todos los participantes con independencia de presentar lesión o no.

AGHO: ácidos grasos hiperoxigenados; PCNl: protector cutáneo no irritante. 
J.E. Torra-Bou, J.J. Soldevilla-Agreda, F.P. García-Fernández, J. Verdú-Soriano, M. Rodríguez-Palma, J.F. Jiménez-García, J.A. Esperón-Güimil, C. Blasco-García, P. Ibars-Moncasí, J. Rueda-López,

E. Perdomo-Pérez, T. Segovia-Gómez. L. Arantón-Areosa, P. López-Casanova, F. Martínez-Cuervo y P.L Pancorbo-Hidalgo

Incidencia, tipología y medidas de prevención y tratamiento de las lesiones cutáneas asociadas al uso de EPI en profesionales sanitarios durante la pandemia de COVID-19 en España

\section{Incidencia de LC-EPI}

Más de la mitad de los respondedores (1088; 52,4\%) manifestaron haber presentado al menos una LC-EPI, por lo que la incidencia de LCEPI en nuestra muestra fue del $52,4 \%$. De acuerdo con la variable sexo, un $54,1 \%$ de las mujeres presentaron LC-EPI frente a un $42,8 \%$ en los hombres.

En las tablas 4, 5 y 6 se resume la información relativa a los profesionales incidentes de LC-EPI según sexo, ámbito asistencial y profesión.

\section{Descripción de las LC-EPI}

Las 1088 personas que presentaron LC-EPI reportan datos de 1387 lesiones con una media de 1,54 $\pm 0,93$ (DE) dispositivos causantes (IC 95\%: 1,54; 1,64).

El 74,3\% de las lesiones fueron LPP, el 11,3\% lesiones por fricción, el $8,5 \%$ lesiones combinadas o multicausales y el $6,1 \%$ restantes lesiones cutáneas asociadas a la humedad.

Las lesiones estuvieron activas una media de 11,63 $\pm 18,02$ (DE) días (IC95\% media: 10,67; 12,59).
En las tablas 7 a 9 y las figuras 4 a 6 se resume la información relativa a la severidad y duración de las lesiones según el tipo de lesión, así como sus localizaciones, dispositivo causante y tiempo de utilización del dispositivo.

\section{DISCUSIÓN}

En nuestro estudio, la metodología utilizada, un cuestionario en línea Google Forms, ha demostrado ser de gran utilidad para estudios con grandes ámbitos poblacionales, ya que ha facilitado llegar a un número elevado de profesionales de la salud utilizando las redes sociales y cumple plenamente con las expectativas de su uso en investigación de la salud en consonancia con lo descrito por Mondal et al. en $2018^{26}$. Además, esta metodología nos ha permitido recabar información complementaria, así como recibir retroalimentación acerca del estudio.

La muestra estudiada, de más de dos mil profesionales, y la distribución por profesiones y niveles asistenciales sitúan al presente estudio entre los de mayor número de participantes, y en nuestro caso, con una amplia representatividad territorial, de niveles asistenciales y de profesiones sanitarias.

Tabla 4. Datos sobre los profesionales incidentes de lesiones cutáneas

\begin{tabular}{|c|c|c|c|}
\hline \multirow{3}{*}{ Total } & Todos (de 18 a 67 años) & Mujeres (de 19 a 66 años) & Hombres (de 18 a 67 años) \\
\hline & $\begin{array}{l}\text { Media [DE] } \\
\text { (IC 95\%) }\end{array}$ & $\begin{array}{l}\text { Media [DE] } \\
\text { (IC 95\%) }\end{array}$ & $\begin{array}{l}\text { Media [DE] } \\
\text { (IC 95\%) }\end{array}$ \\
\hline & $\begin{array}{c}43,7[10,8 \text { años }] \\
(43,2 ; 44,1)\end{array}$ & $\begin{array}{c}43,4[10,7 \text { años }] \\
(42,9 ; 43,9)\end{array}$ & $\begin{array}{c}45,4 \text { [11,1 años] } \\
(44,2 ; 46,7)\end{array}$ \\
\hline N. ${ }^{0}$ de lesiones & $\begin{array}{l}39 \% \text { una lesión } \\
35,8 \% \text { dos lesiones } \\
14,3 \% \text { tres lesiones } \\
2,2 \% \text { cuatro lesiones } \\
\text { 8,6\% más de cuatro lesiones }\end{array}$ & $\begin{array}{l}39 \% \text { una lesión } \\
34,9 \% \text { dos lesiones } \\
14,9 \% \text { tres lesiones } \\
2,4 \% \text { cuatro lesiones } \\
\text { 8,8\% más de cuatro lesiones }\end{array}$ & $\begin{array}{l}39 \% \text { una lesión } \\
42,6 \% \text { dos lesiones } \\
10,3 \% \text { tres lesiones } \\
\text { 0,7\% cuatro lesiones } \\
7,3 \% \text { más de cuatro lesiones }\end{array}$ \\
\hline
\end{tabular}

Tabla 5. Incidencia de LC-EPI y número de lesiones en profesionales de la salud según ámbito asistencia

\begin{tabular}{|l|c|c|c|c|c|}
\hline & Todos & Hospitales & Atención primaria & $\begin{array}{c}\text { Atención } \\
\text { sociosanitaria }\end{array}$ & $\begin{array}{c}\text { Atención extrahospitalaria } \\
\text { urgente }\end{array}$ \\
\hline Incidencia de LC-EPI & $52,4 \%$ & $58,5 \%$ & $36,9 \%$ & $47,7 \%$ & $47,6 \%$ \\
\hline 1 lesión & $39 \%$ & $70,9 \%$ & $47,8 \%$ & $44,4 \%$ & $40 \%$ \\
\hline 2 lesiones & $35,8 \%$ & $36,8 \%$ & $30,9 \%$ & $38,9 \%$ & $10 \%$ \\
\hline 3 lesiones & $14,3 \%$ & $15,3 \%$ & $11,2 \%$ & $11,1 \%$ & $10 \%$ \\
\hline 4 lesiones & $2,2 \%$ & $2,3 \%$ & $1,7 \%$ & $2,8 \%$ & 0 \\
\hline
\end{tabular}

Tabla 6. Incidencia de LC-EPI y número de lesiones según profesión

\begin{tabular}{|l|c|c|c|c|c|c|}
\hline & Todos & Enfermera/0 & TCAE & Médico/a & Fisioterapeuta & Celador \\
\hline Incidencia de LC-EPI & $52,4 \%$ & $51,6 \%$ & $62,8 \%$ & $50,4 \%$ & $53,8 \%$ & $30,4 \%$ \\
\hline 1 lesión & $39 \%$ & $39,7 \%$ & $31,4 \%$ & $43,5 \%$ & $35,7 \%$ & $28,6 \%$ \\
\hline 2 lesiones & $35,8 \%$ & $36,2 \%$ & $34,3 \%$ & $36,2 \%$ & $35,7 \%$ & $28,6 \%$ \\
\hline 3 lesiones & $14,3 \%$ & $13,5 \%$ & $20,7 \%$ & $14,5 \%$ & $14,3 \%$ & $28,6 \%$ \\
\hline 4 lesiones & $2,2 \%$ & $2,7 \%$ & $0,7 \%$ & 0 & $7,1 \%$ & 0 \\
\hline Más de 4 lesiones & $8,6 \%$ & $7,9 \%$ & $12,9 \%$ & $5,8 \%$ & $7,1 \%$ & $14,3 \%$ \\
\hline
\end{tabular}


Tabla 7. Severidad/profundidad de las LC-EPI según tipo de lesión

\begin{tabular}{|c|c|c|c|}
\hline $\begin{array}{l}\text { Lesión por presión } \\
\text { (967; 74,3\% del total) }\end{array}$ & $\begin{array}{l}\text { Lesión por fricción } \\
\text { (144; 11\% del total) }\end{array}$ & $\begin{array}{l}\text { Lesión combinada o multicausal } \\
\text { (111; } 8,5 \% \text { del total) }\end{array}$ & $\begin{array}{c}\text { Lesión cutánea asociada a la } \\
\text { humedad } \\
\text { (80; 6,1\% del total) }\end{array}$ \\
\hline Categoría I: 600 & Categoría I: 102 & Categoría I: 48 & Categoría 1A: 34 \\
\hline Categoría II: 341 & Categoría II: 24 & Categoría II: 59 & Categoría 1 B: 33 \\
\hline \multirow[t]{2}{*}{ Categoría III: 26} & Categoría III: 18 & Categoría III: 3 & Categoría 2 A: 13 \\
\hline & & Categoría IV: 1 & \\
\hline $\begin{array}{c}\bar{x}=9,7 \text { (DE: 15,6) días de duración } \\
\text { IC95\%: }(8,7 ; 10,7) \\
\text { Mín.: } 1 \text { día; Máx.: } 100 \text { días }\end{array}$ & $\begin{array}{c}\bar{x}=10,2 \text { (DE: 15,7) días de duración } \\
\text { IC95\%: }(7,6 ; 12,8) \\
\text { Mín.: } 1 \text { día; Máx.: } 100 \text { días }\end{array}$ & $\begin{array}{c}\bar{x}=19,9 \text { (DE: 26,3) días de duración } \\
\text { IC95\%: }(15 ; 24,9) \\
\text { Mín.: } 1 \text { día; Máx:: } 90 \text { días }\end{array}$ & $\begin{array}{c}\bar{x}=19,4 \text { (DE: 22,4) días de duración } \\
\text { IC 95\%: }(15 ; 23,9) \\
\text { Mín.: } 1 \text { día; Máx.: } 90 \text { días }\end{array}$ \\
\hline
\end{tabular}

Tabla 8. Localizaciones de las LC-EPI según tipo de lesión

\begin{tabular}{|c|c|c|c|c|c|c|c|c|c|c|}
\hline \multirow[b]{2}{*}{ Localizaciones } & \multicolumn{2}{|c|}{ Todas las lesiones } & \multicolumn{2}{|r|}{ LPP } & \multicolumn{2}{|c|}{ Multicausales } & \multicolumn{2}{|r|}{ Fricción } & \multicolumn{2}{|r|}{ LESCAH } \\
\hline & $\mathrm{n}$ & $\begin{array}{l}\text { Porcentaje sobre } \\
\text { el total de lesiones }\end{array}$ & $\mathrm{n}$ & $\begin{array}{l}\text { Porcentaje sobre } \\
\text { el total de lesiones }\end{array}$ & $\mathrm{n}$ & $\begin{array}{l}\text { Porcentaje sobre } \\
\text { el total de lesiones }\end{array}$ & $\mathrm{n}$ & $\begin{array}{l}\text { Porcentaje sobre } \\
\text { el total de lesiones }\end{array}$ & $\mathrm{n}$ & $\begin{array}{l}\text { Porcentaje sobre } \\
\text { el total de lesiones }\end{array}$ \\
\hline Cabeza/cuero cabelludo & 12 & 0,37 & 6 & 0,18 & 3 & 0,09 & 1 & 0,03 & 2 & 0,06 \\
\hline Sienes & 1 & 0,03 & & 0,00 & & 0,00 & 1 & 0,03 & & 0,00 \\
\hline Frente & 347 & 10,61 & 289 & 8,84 & 14 & 0,43 & 35 & 1,07 & 9 & 0,28 \\
\hline Orejas & 623 & 19,06 & 540 & 16,52 & 25 & 0,76 & 47 & 1,44 & 11 & 0,34 \\
\hline Área sellado gafas protectoras & 191 & 5,84 & 148 & 4,53 & 16 & 0,49 & 14 & 0,43 & 13 & 0,40 \\
\hline Ojos/estructuras oculares & 6 & 0,18 & 3 & 0,09 & 3 & 0,09 & & 0,00 & & 0,00 \\
\hline Puente de la nariz & 902 & 27,59 & 770 & 23,55 & 48 & 1,47 & 63 & 1,93 & 21 & 0,64 \\
\hline Punta de la nariz & 97 & 2,97 & 78 & 2,39 & 5 & 0,15 & 11 & 0,34 & 3 & 0,09 \\
\hline Contorno nariz & 4 & 0,12 & 3 & 0,09 & & 0,00 & & 0,00 & 1 & 0,03 \\
\hline Alas nariz & 4 & 0,12 & 2 & 0,06 & & 0,00 & 1 & 0,03 & 1 & 0,03 \\
\hline Fosas nasales & 1 & 0,03 & & 0,00 & 1 & 0,03 & & 0,00 & & 0,00 \\
\hline Entre nariz y boca & 1 & 0,03 & 1 & 0,03 & & 0,00 & & 0,00 & & 0,00 \\
\hline Pómulos & 307 & 9,39 & 253 & 7,74 & 20 & 0,61 & 20 & 0,61 & 14 & 0,43 \\
\hline Mejillas & 373 & 11,41 & 300 & 9,18 & 26 & 0,80 & 23 & 0,70 & 24 & 0,73 \\
\hline Peribucal/labios & 14 & 0,43 & 8 & 0,24 & 2 & 0,06 & & 0,00 & 4 & 0,12 \\
\hline Barbilla/mentón & 62 & 1,90 & 27 & 0,83 & 9 & 0,28 & 9 & 0,28 & 17 & 0,52 \\
\hline Garganta/Cuello & 54 & 1,65 & 33 & 1,01 & 2 & 0,06 & 4 & 0,12 & 5 & 0,15 \\
\hline Nuca & 1 & 0,03 & & 0,00 & & 0,00 & 1 & 0,03 & & 0,00 \\
\hline Espalda & 2 & 0,06 & 2 & 0,06 & & 0,00 & & 0,00 & 1 & 0,03 \\
\hline Tronco & 4 & 0,12 & & 0,00 & & 0,00 & 1 & 0,03 & 3 & 0,09 \\
\hline Zona sacra & 1 & 0,03 & & 0,00 & & 0,00 & & 0,00 & 1 & 0,03 \\
\hline Brazo/antebrazo & 12 & 0,37 & 5 & 0,15 & 2 & 0,06 & 2 & 0,06 & 3 & 0,09 \\
\hline Muñecas & 4 & 0,12 & 3 & 0,09 & & 0,00 & & 0,00 & 1 & 0,03 \\
\hline Manos & 242 & 7,40 & 162 & 4,96 & 27 & 0,83 & 22 & 0,67 & 31 & 0,95 \\
\hline Muslo & 2 & 0,06 & 2 & 0,06 & & 0,00 & & 0,00 & & 0,00 \\
\hline Dedo mano & 1 & 0,03 & & 0,00 & & 0,00 & & 0,00 & 1 & 0,03 \\
\hline Dedos pie & 1 & 0,03 & & 0,00 & & 0,00 & 1 & 0,03 & & 0,00 \\
\hline TOTAL & 3269 & 100,00 & 2635 & 80,61 & 203 & 6,21 & 256 & 7,83 & 166 & 5,08 \\
\hline
\end{tabular}


J.E. Torra-Bou, J.J. Soldevilla-Agreda, F.P. García-Fernández, J. Verdú-Soriano, M. Rodríguez-Palma, J.F. Jiménez-García, J.A. Esperón-Güimil, C. Blasco-García, P. Ibars-Moncasí, J. Rueda-López,

E. Perdomo-Pérez, T. Segovia-Gómez, L. Arantón-Areosa, P. López-Casanova, F. Martínez-Cuervo y P.L Pancorbo-Hidalgo

Incidencia, tipología y medidas de prevención y tratamiento de las lesiones cutáneas asociadas al uso de EPI en profesionales sanitarios durante la pandemia de COVID-19 en España

Tabla 9. Dispositivo causante de la LC-EPI

\begin{tabular}{|c|c|c|c|c|c|c|c|c|c|c|}
\hline & \multicolumn{2}{|c|}{ Todas } & \multicolumn{2}{|c|}{ LPP } & \multicolumn{2}{|c|}{ Multicausales } & \multicolumn{2}{|c|}{ Fricción } & \multicolumn{2}{|c|}{ LESCAH } \\
\hline & $n$ & $\%$ & $n$ & $\%$ & $n$ & $\%$ & $n$ & $\%$ & $\mathrm{n}$ & $\%$ \\
\hline Mascarilla quirúrgica & 248 & $12 \%$ & 153 & $10,4 \%$ & 30 & $17,5 \%$ & 24 & $12,1 \%$ & 28 & $18,4 \%$ \\
\hline Mascarilla FFP2 & 630 & $30,4 \%$ & 464 & $31,5 \%$ & 52 & $30,4 \%$ & 62 & $31,2 \%$ & 34 & $22,4 \%$ \\
\hline Mascarilla FFP3 & 214 & $10,3 \%$ & 168 & $11,4 \%$ & 10 & $3,8 \%$ & 18 & $9 \%$ & 12 & $7,9 \%$ \\
\hline Mascarilla KN95 & 238 & $11,5 \%$ & 183 & $12,9 \%$ & 17 & $9,9 \%$ & 21 & $10,6 \%$ & 10 & $6,6 \%$ \\
\hline Gafas protección & 412 & $19,9 \%$ & 325 & $22,1 \%$ & 24 & $14 \%$ & 35 & $17,6 \%$ & 14 & $9,2 \%$ \\
\hline Pantalla protectora & 118 & $5,7 \%$ & 82 & $5,6 \%$ & 9 & $5,3 \%$ & 17 & $8,5 \%$ & 7 & $4,6 \%$ \\
\hline Guantes y geles hidroalcohólicos & 109 & $5,3 \%$ & 46 & $3,1 \%$ & 19 & $11,1 \%$ & 11 & $5,5 \%$ & 26 & $17,1 \%$ \\
\hline Gorro & 36 & $1,7 \%$ & 26 & $1,8 \%$ & 1 & $0,6 \%$ & 2 & $1 \%$ & 4 & $2,6 \%$ \\
\hline Bata & 24 & $1,2 \%$ & 11 & $0,7 \%$ & 2 & $1,2 \%$ & 3 & $1,5 \%$ & 5 & $3,3 \%$ \\
\hline Buzo completo & 32 & $2,8 \%$ & 13 & $0,9 \%$ & 5 & $2,9 \%$ & 5 & $2,5 \%$ & 8 & $5,3 \%$ \\
\hline Delantal & 6 & $0,3 \%$ & 1 & $0,1 \%$ & - & - & - & - & 4 & $2,6 \%$ \\
\hline Ropa descartable & 1 & $0,05 \%$ & 1 & $0,1 \%$ & - & - & - & - & - & - \\
\hline Adhesivo para fijar KN95 & 2 & $0,1 \%$ & - & - & 2 & $1,2 \%$ & - & - & - & - \\
\hline Zapatos & 1 & $0,05 \%$ & - & - & - & - & 1 & $0,5 \%$ & - & - \\
\hline
\end{tabular}

En 76 de las lesiones no se describe el dispositivo causante.

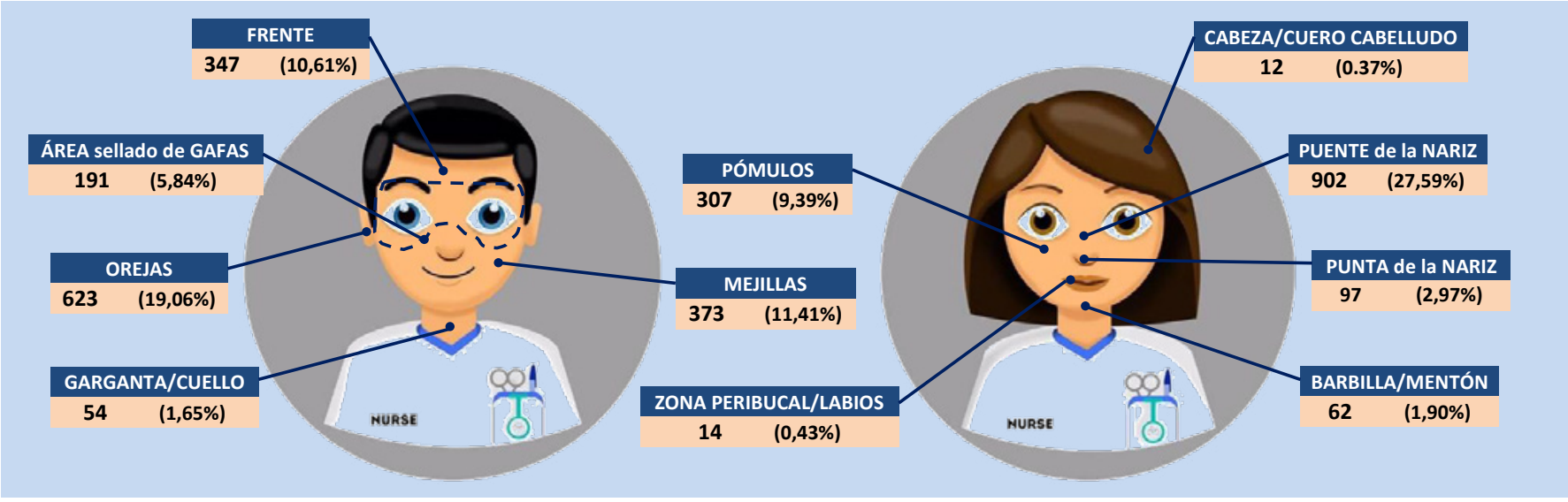

Figura 4. Localización del total de LC- EPI a nivel de cara y cuello (se expresa en número de lesiones y porcentaje sobre el total).

Fuente imagen: Campaña \#NurseEmoji

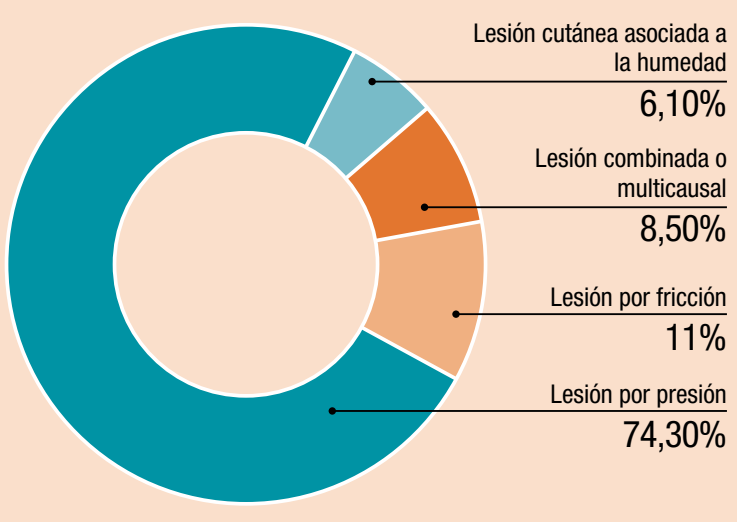

Figura 5. Tipos de lesiones.
La edad media de los profesionales respondedores nos sitúa ante un colectivo de profesionales de la salud de edad madura con unos 20-25 años de ejercicio profesional.

En cuanto a la disponibilidad de protocolo de prevención de LC-EPI en las instituciones, destaca que solo una quinta parte de las instituciones contaban con dicho protocolo, y que uno de cada diez profesionales no sabía si su institución contaba con protocolo o no. El ámbito asistencial con menor disponibilidad de protocolo es la atención primaria de salud.

En paralelo a la elaboración del presente informe de investigación, y tras un primer análisis preliminar de los resultados de la presente investigación, desde el GNEAUPP se ha elaborado un documento técnico sobre prevención de LC-EPI para poder mejorar la protección de los profesionales de la salud ${ }^{27}$, sin lugar a duda segundas víctimas en esta pandemia.

Una circunstancia a destacar es que en un importante porcentaje de casos son los profesionales de la salud quienes tienen que autoproveerse 


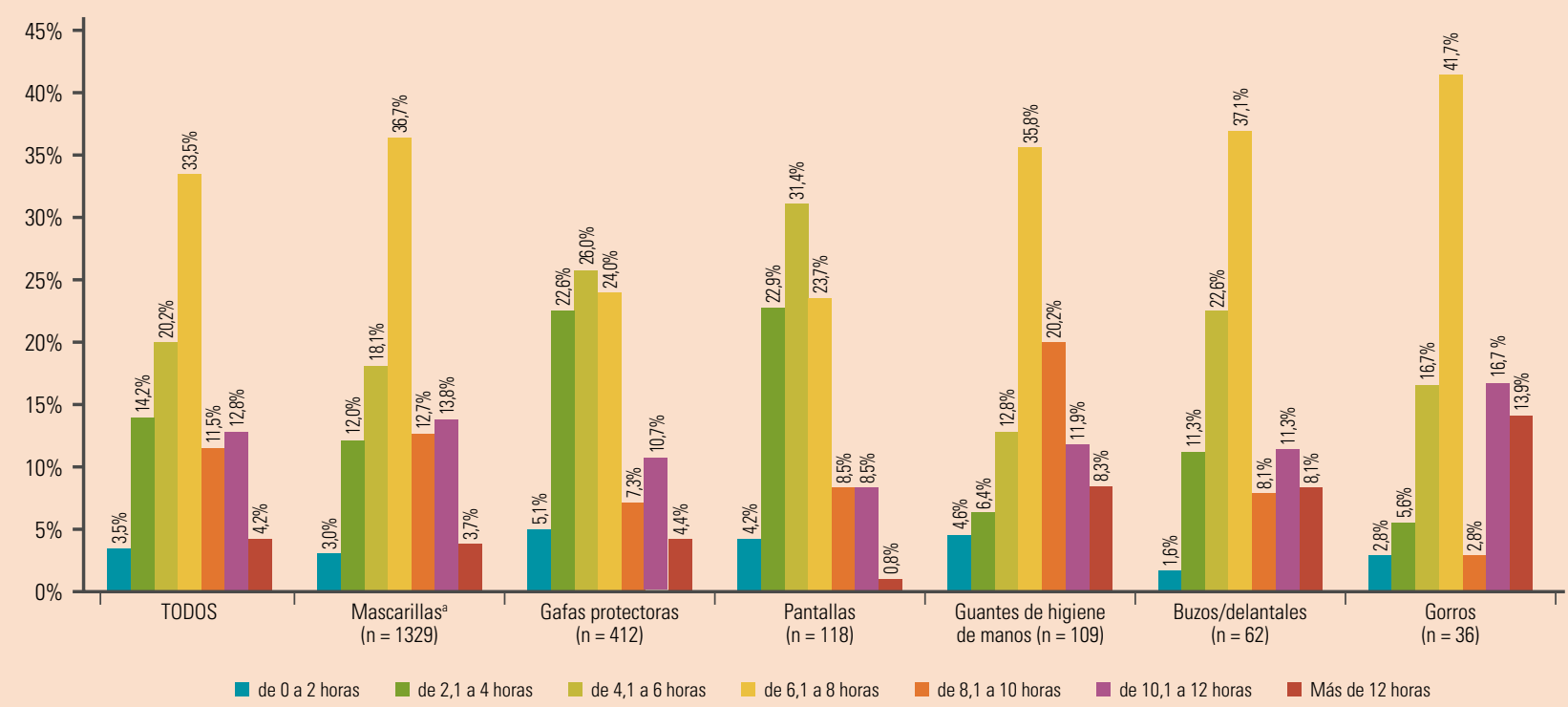

Figura 6. Dispositivo causante y horas al día de utilización.

aQuirúrgicas, FFP2, FFP3 y KN95.

de material de prevención ante las LC-EPI. Disponer de protocolos de prevención y asociar la disponibilidad de recursos específicos de prevención basados en la mejor evidencia posible es, sin lugar a duda, una medida básica para reducir el impacto de las LC-EPI en los profesionales de la salud.

En cuanto a los dispositivos o productos utilizados para la prevención de LC-EPI destacan las cremas y/o aceites hidratantes, los AGHO y las cremas barreras para la protección y/o cuidado de la piel, mientras que para el alivio de la presión o fricción lo hacen los apósitos hidrocoloides y los apósitos de espuma de poliuretano. Llama la atención la utilización de apósitos de gasa/esparadrapo y de películas de poliuretano.

La disparidad de productos utilizados, tanto para la prevención y cuidado de la piel como para la protección ante la presión y fricción, ponen de manifiesto la necesidad de que se investigue acerca de la idoneidad de dichos productos de cara a su efecto protector.

En cuanto a la incidencia de LC-EPI, las cifras encontradas en nuestra muestra tras el primer brote de COVID-19 en España, un 52,4\%, nos sitúan en una cifra alarmante que no nos atrevemos a comparar con la literatura existente debido a la gran disparidad de criterios y enfoques metodológicos utilizados ${ }^{10-24}$. Otro hecho a destacar, y que no ha sido analizado en otros estudios, es que un $60 \%$ de los profesionales incidentes de LC-EPI han presentado más de un episodio. Esta circunstancia resalta la necesidad de definir e instaurar protocolos efectivos de prevención.

En cuanto al tipo de lesión, nuestro estudio aporta a la literatura científica sobre el tema datos sistematizados sobre tipo de lesión, duración y nivel de gravedad, datos que una vez más no podemos comparar con la literatura debido a la disparidad de enfoques y criterios, pero que creemos que puede ser de gran utilidad para dimensionar de manera pormenorizada el impacto y las variables que definen el problema de las LC-EPI.

Un elemento importante a destacar en cuanto a la categorización de las LC-EPI es que aunque las LPP sean el tipo de lesión más frecuente dentro de dicho tipo de lesiones, un $74,3 \%$ en nuestro estudio, existen otros tipos de lesiones englobados bajo el concepto de las LCRD; es el caso de las lesiones por fricción (11\%), combinadas o multicausales $(8,5 \%)$ o las LESCAH $(6,1 \%)$; es por ello por lo que aunque algunos autores ${ }^{28}$ definen a todas las LC-EPI como LPP-DISCLIN, consideramos que estas deberían clasificarse como LCRD, aunque ello obligue a replantear o considerar en este caso el concepto de relacionadas con la dependencia, lo que podría ser una contradicción en el caso de los profesionales de la salud como sujetos que las padecen. Sin lugar a duda, esta disquisición en cuanto a la clasificación y denominación de las LC-EPI abre nuevas líneas de investigación y debate en la comunidad científica dedicada al cuidado de las heridas y de la piel.

En relación con las lesiones y sus repercusiones, dos elementos a destacar: el elevado número de lesiones con rotura de la piel en todos los tipos de lesiones y la duración de los episodios.

En cuanto a las localizaciones de las LC-EPI, las más frecuentes, puente de la nariz, orejas, mejillas, frente, pómulos, manos y área de sellado de gafas protectoras, concuerdan con las identificadas en la literatura.

Nuestro estudio también aporta información acerca de la relación entre dispositivo causante y tipo de lesión, así como las horas de utilización de los dispositivos. Esta información es muy importante para definir protocolos de prevención de LC-EPI basándose en la dinámica de utilización de los mismos

\section{Conflicto de intereses}

Ninguno de los autores manifiesta tener conflicto de interés alguno en relación con la temática de la investigación.

\section{Agradecimientos}

El equipo investigador quiere dar las gracias a los más de 2000 profesionales de la salud que han participado en el estudio, así como a las sociedades científicas, instituciones y organizaciones profesionales que han colaborado en la difusión del estudio. De la misma manera, queremos agradecer la gran cantidad de mensajes de apoyo y retroalimentación recibidos por parte de los participantes en el estudio. 
J.E. Torra-Bou, J.J. Soldevilla-Agreda, F.P. García-Fernández, J. Verdú-Soriano, M. Rodríguez-Palma, J.F. Jiménez-García, J.A. Esperón-Güimil, C. Blasco-García, P. Ibars-Moncasí, J. Rueda-López, E. Perdomo-Pérez, T. Segovia-Gómez, L. Arantón-Areosa, P. López-Casanova, F. Martínez-Cuervo y P.L Pancorbo-Hidalgo

Incidencia, tipología y medidas de prevención y tratamiento de las lesiones cutáneas asociadas al uso de EPI en profesionales sanitarios durante la pandemia de COVID-19 en España

\section{У BIBLIOGRAFÍA}

1. Tomás-Vecina S, Martín-Delgado MC, Mingueza Sebastián Y, Fuz F, Gómez Felipe E, Benet J. Seguridad del paciente y profesional durante la epidemia SARS-COV-2 (COVID-19) en España. Proyecto SEGCOVID. Fundación para la investigación, docencia e investigación en Seguridad del Paciente y Saham. Disponible en: https://fidisp.org/wp-content/uploads/2020/10/PROYECTO-SEGCOVID-DEFINITIVO.pdf (acceso 29-11-2020)

2. Torra-Bou JE. Constatando con cifras la epidemia de lesiones por presión como consecuencia de la primera fase de la pandemia de COVID-19 en España. [Internet]. Blog Piel Segura. 2020 [citado 29 de noviembre de 2020]. Accesible en: http:// sapiensjetorrabou.com/constatando-con-cifras-la-epidemia-delesiones-por-presion-como-consecuencia-de-la-primera-fasede-la-pandemia-de-covid-19-en-espana/

3. Plan de Calidad para el Sistema Nacional de Salud. Ministerio de Sanidad y Consumo. Estudio nacional sobre los efectos adversos ligados a la hospitalización. ENEAS 2005. Informe Febrero 2006. Madrid: Ministerio de Sanidad y Consumo; 2006

4. García-Fernández FP, Soldevilla-Agreda JJ, Pancorbo-Hidalgo PL, Verdú-Soriano J, López-Casanova P, Rodríguez-Palma M. Clasificación-categorización de las lesiones relacionadas con la dependencia. Serie Documentos Técnicos GNEAUPP n 11 . Grupo Nacional para el Estudio y Asesoramiento en Úlceras por Presión y Heridas Crónicas. Logroño, 2014.

5. García-Fernández FP, Agreda JJ, Verdú J, Pancorbo-Hidalgo PL. A New Theoretical Model for the Development of Pressure Ulcers and Other Dependence-Related Lesions. J Nurs Scholarsh. 2014:46(1):28-38. doi: 10.1111/jnu.12051. Epub 2013 Oct 11

6. David SJ. History in a Crisis - Lessons for Covid-19. N Engl J Med 2020:382:1681-3 doi: 10.1056/NEJMp2004361

7. Fletcher J. Device related pressure ulcers made easy. Wounds UK. 2012;8(2):1-4.

8. Edsberg LE, Black JM, Goldberg M, McNichol L, Moore L Sieggreen M. Revised National Pressure Ulcer Advisory Panel pressure injury staging system: revised pressure injury staging system. J Wound Ostomy Continence Nurs. 2016;43(6):585.

9. Black JM, Kalowes P. Medical device-related pressure ulcers. Chronic Wound Care Management and Research 2016;3:91-99.
10. Gefen A, Alves P, Ciprandi G, Coyer F, Milne CT, Ousey K, et al. Device related pressure ulcers: SECUREprevention. J Wound Care. 2020;29(Sup2a):S1-52.

11. Mazzotta F, Troccoli T. 2020. Acute acro-ischemia in the child at the time of COVID-19. Eur J Pediat Dermatol. 2020:30(2):71-4. doi: $10.26326 / 2281-9649.30$

12. Zhang Y, Cao W, Xiao M, Li YJ, Yang Y, Zhao J, et al. 2020. Clinical and Coagulation Characteristics of 7 Patients With Critical COVID-2019 Pneumonia and Acro-Ischemia. Zhonghua Xue Ye Xue Za Zhi. 2020;(0): E006 [Online ahead of print]. doi: 10.3760/ cma.j.issn.0253-2727.2020.0006

13. Recalcati S. Cutaneous Manifestations in COVID-19: A First Perspective. Eur Acad Dermatol Venereol. 2020 Mar 26 [Online ahead of print].

14. Estébanez A Pérez-Santiago L Silva E Gullien-Climent $S$, García-Vázquez A, Ramón MD. Cutaneous Manifestations in COVID-19: A New Contribution. J Eur Acad Dermatol Venereol. 2020;34(6):e250-51. doi: 10.1111/jdv.16474

15. Jiang 0 , Song $S$, Zhou J, Liu Y, Chen A, Bai Y, et al. The Prevalence, Characteristics, and Prevention Status of Skin Injury Caused by Personal Protective Equipment Among Medical Staff in Fighting COVID-19: A Multicenter, Cross-Sectional Study. Adv Wound Care (New Rochelle). 2020;9(7):357-64. doi: 10.1089/ wound.2020.1212. Epub 2020 Apr 27

16. Pei S, Xue Y, Zhao S, Alexander N, Mohamand G, Chen X, et al. Occupational skin conditions on the front line: a survey among 484 Chinese healthcare professionals caring for Covid-19 patients. J Eur Acad Dermatol Venereol. 2020;34(8):e354-7. doi: 10.1111/jdv.16570. Epub 2020 Jun 8

17. Lan J, Song Z, Miao X, Li H, Li Y, Dong L, et al. Skin damage among healthcare workers managing coronavirus disease-2019, J Am Acad Dermatol. 2020;82(5):1215-6. doi: https://doi.org/10.1016/j.jaad.2020.03.014

18. P. Lin S, Zhu Y, Huang L, Li L, Tao J, Lei T, et al. Adverse Skin Reactions Among Healthcare Workers During the Coronavirus Disease 2019 Outbreak: A Survey in Wuhan and Its Surrounding Regions. Br J Dermatol. 2020. https://doi.org/10.1111/bjd.19089

19. Altinusik S, Altunisik N, Turkmen D, Ersoy Y. Relationship between hand hygiene and cutaneous findings during COVID-19 pandemic. J Cosmetic Dermatol. 2020. https://doi.org/10.1111/jocd.13656
20. Hadjieconomou S, Hughes J, Kamath S. Occupational skin disease during the COVID-19 pandemic, as captured in a Dermatology staff clinic in the United Kingdom. J Eur Acad Dermatol Venereol. 2020;34(11):e670-1. doi: 10.1111/jdv.16754

21. Hu K, Fan J, LiX Gou X, Li X, Zhou X. The adverse skin reaction of health care workers using personal protective equipment for COVID-19. Medicine. 2020;99:24(e20603)

22. Malivar K, Sachdeva M, Mufti A, Yeung J. Reply to:"Skin damage among health care workers managing coronavirus disease 2019". J Am Acad Dermatol. 2020;83(2):169-70.

23. Hu D, Kong Y, Li W, Han Q, Zhang X, Zhu LX, et al. Frontline nurses, burnout, anxiety, depression and fear status and their associated factors during the COVID-19 outbreak in Wuhan China: A large scale cross-sectional study. EClinicalMedicine. 2020:24:100424. https://doi.org/10.1016/j.eclinm.2020.100424

24. 24. Shaukat N, Mansoor D, Razzak J. Physical and mental health impacts of COVID-19 on healthcare workers: a scoping review. Int J Emerg Med. 202013:40. https://doi.org/10.1186/s12245020-00299-5

25. Reglamento (UE) 2016/679 del Parlamento Europeo y del Consejo de 27 de abril de 2016, relativo a la protección de las personas fisicas en lo que respecta al tratamiento de datos personales y a la libre circulación de estos datos y por el que se deroga la Directiva 95/46/CE (Reglamento general de protección de datos).

26. Mondal H, Moldal H Mondal S, Ghosal T, Mondal S. Using Google Forms for Medical Survey: A Technical Note. Int J Clin Exp Physiol. 2018;5(4).

27. Rueda López J, Blasco García C, Segovia Gómez T, Martínez Cuevvo F Perdomo Pérez E llars Moncasí P. Torra i Bou JE. Prevención de las lesiones cutáneas relacionadas con la utilización de equipos de protección individual en profesionales sanitarios: Guía de recomendaciones. Serie Documentos de Posicionamiento GNEAUPP $n^{0} 16$. Grupo Nacional para el Estudio y Asesoramiento en Úlceras por Presión y Heridas Crónicas. Logroño, 2020.

28. Yıldız A, Karadağ A, Yıldız A, Cakar V. Determination of the effect of prophylactic dressing on the prevention of skin injuries associated with personal protective equipments in health care workers during COVID-19 pandemic. J Tissue Viability. 2020 Nov 1: S0965-206X(20)30125-X. doi: 10.1016/j.jtv.2020.10.005. Epub ahead of print. PMID: 33187790. 